\title{
RESEARCH
}

Open Access

\section{Roles of NFkB-miR-29s-MMP-2 circuitry in experimental choroidal neovascularization}

Jingjing Cai ${ }^{1,2+}$, Guibin Yin ${ }^{1,2 \dagger}$, Bing Lin ${ }^{1,2}$, Xianwei Wang ${ }^{1,2}$, Xiaoling Liu ${ }^{1,2}$, Xiaoyan Chen ${ }^{1,2}$, Dongsheng Yan ${ }^{1,2}$, Ge Shan ${ }^{3}$, Jia Qu $u^{1,2}$ and Shengzhou Wu $\mathrm{u}^{1,2^{*}}$

\begin{abstract}
Background: Previous reports have indicated that matrix metallopeptidase-2 (MMP-2) regulates angiogenic processes, which are involved in choroidal neovascularization (CNV). However, the regulation of MMP-2 in CNV has not been well-characterized. To gain more information about the regulation of MMP-2 in CNV, we analyzed the circuitry associated with MMP-2 regulation in a CNV model and in cell cultures, focusing on NFKB and the microRNA-29 family (miR-29s).
\end{abstract}

Methods: The CNV model was established by subjecting C57BL/6 mice to fundus photocoagulation with a krypton red laser. In choroidal-retinal pigment epithelial (RPE) tissues of the model, immunohistochemistry was used to evaluate the angiogenesis and MMP-2 expression; reverse-transcription quantitative PCR (RT-qPCR) was used to determine the levels of miR-29s; and western blot was used to analyze the protein levels of nuclear factor kappa-light-chainenhancer of activated B cells (NFKB) inhibitor, IKBa, and its phosphorylated form, phospho-IKBa. At the cellular level, RT-qPCR was used to examine the levels of miR-29s following NFKB activation by tumor necrosis factor alpha (TNFa); and western blot and luciferase assay were used to determine the regulation of MMP-2 by miR-29s in a human RPE cell line (ARPE-19) and in an umbilical vein endothelial cell line (EA hy926).

Results: MMP-2 staining was increased in the choroidal neovascular membrane of laser-treated retina. Also, the NFKB pathway was induced in choroid-RPE tissue, as evidenced by a lower protein level of $\mathrm{k}_{\mathrm{K} B a}$ and a higher level of phospho-IkBa in the tissue homogenates than in those from non-treated eyes. During the period when the NFKB pathway was induced, reduced miR-29s were detected in the choroidal-RPE tissue of the laser-treated eyes. In cultured ARPE-19 cells, TNFa decreased miR-29a, b, and c, and the effects were rescued by NFKB decoy. In ARPE-19 and EA hy926, miR-29s mimics reduced the contents of secreted MMP-2 in the culture media. We also documented that miR-29s reduced MMP-2 3'-UTR-mediated luciferase transcription.

Conclusions: The results suggest that in CNV, NFKB activation inhibits miR-29s, which may contribute to angiogenesis by up-regulating the MMP-2 protein level in RPE cells. These observations may help in developing a strategy for resolving CNV by targeting miR-29s levels.

Keywords: Choroidal neovascularization (CNV), Matrix metallopeptidase-2 (MMP-2), Nuclear factor kappa-lightchain-enhancer of activated B cells (NFkB), microRNA-29 family (miR-29s), Tumor necrosis factor alpha (TNFa)

\footnotetext{
*Correspondence: shzhwu1@gmail.com

${ }^{\dagger}$ Equal contributors

${ }^{1}$ School of Optometry and Ophthalmology and Eye Hospital, Wenzhou

Medical University, 270 Xueyuan Road, Wenzhou, Zhejiang 325003, People's

Republic of China

${ }^{2}$ State Key Laboratory Cultivation Base and Key Laboratory of Vision Science,

Ministry of Health, China and Zhejiang Provincial Key Laboratory of

Ophthalmology and Optometry, 270 Xueyuan Road, Wenzhou, Zhejiang

325003, People's Republic of China

Full list of author information is available at the end of the article
}

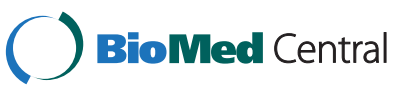

(C) 2014 Cai et al.; licensee BioMed Central Ltd. This is an Open Access article distributed under the terms of the Creative Commons Attribution License (http://creativecommons.org/licenses/by/2.0), which permits unrestricted use, distribution, and reproduction in any medium, provided the original work is properly credited. The Creative Commons Public Domain Dedication waiver (http://creativecommons.org/publicdomain/zero/1.0/) applies to the data made available in this article unless otherwise stated. 


\section{Introduction}

Choroidal neovascularization (CNV) occurs in wet-type age-related macular degeneration, extreme myopia, and pathological myopia [1-3]. Angiogenesis in CNV is a complicated process with imbalance in the production of pro-angiogenic and anti-angiogenic factors (for example, excessive production of vascular endothelial growth factor (VEGF)), leading to pathological neovascularization [4]. The process of angiogenesis as it occurs in CNV involves the proteolysis of an extracellular matrix, the proliferation and migration of endothelial cells, and the synthesis of a new matrix in the destination [5].

Matrix metalloproteinase (MMP) family proteins play important roles in angiogenesis partly due to their ability to degrade basal membrane and extracellular matrix proteins. MMPs are secreted as zymogens, which require cleavage to create active forms of the enzyme. For instance, matrix metalloproteinase-2 (MMP-2) is derived from proMMP-2 by MT1-MMP cleavage and MMP-9 is derived from pro-MMP9 by MMP3 cleavage [6]. The activity of MMP is also down-regulated by endogenous inhibitors, namely tissue inhibitors of metalloproteinase [7]. Recent studies indicate that MMP-2 is indispensable in forming $\mathrm{CNV}$ because reduced $\mathrm{CNV}$ or attenuated retinal angiogenesis are observed in MMP-2 deficient mice [8,9]. RPE is a major resource for producing MMP-2 in the retina and overexpression of VEGF and MMP-2 by RPE is critical for angiogenesis in CNV [10,11].

MicroRNAs (miRNAs) are a family of endogenous, non-coding small RNAs with mature-type sizes ranging from 19 to 25 nucleotides [12]. In eukaryotes, miRNAs bind to the complementary sequences of the targeted mRNA, mostly 3'-untranslated region (3'-UTR), leading to translation repression or sometimes mRNA degradation [12]. Approximately $50 \%$ of miRNAs constitute clusters in a chromosome and transcribe as polycistronic primary transcripts [13]. Specifically, the microRNA-29 family (miR-29s) consists of a miR-29a/b1 cluster in one chromosome and a miR-29b2/c cluster in a different chromosome. Recent data indicate that miR-29s have multiple functions via binding to the 3'UTR of the target mRNAs: regulation of methylation status in lung cancer via targeting DNA methyltransferases $3 \mathrm{~A}$ and $3 \mathrm{~B}$ [14]; suppression of tumor angiogenesis, invasion and metastasis by regulating MMP-2 expression [15]; regulation of the processing of the $\beta$-amyloid precursor protein by decreasing $\beta$-secretase expression [16]; and activation of p53 via suppressing p85 alpha and CDC42, which both negatively regulate p53 [17]. Regulation of miR-29s also occurs in different ways: NFkB-YY1 pathway activation regulates skeletal muscle differentiation via inhibition of miR-29s transcriptions [18], and miR-29s is up-regulated in a p53-dependent manner in circumstances of aging and chronic DNA damage [19].
Recent studies have suggested that NFKB plays important roles in the early stage of angiogenesis [20,21], in CNV [22]; the inhibition of miR-29s by NFkB occurs in various cells $[18,23]$; and MMP-2 clearly contributes to angiogenesis [5]. These lines of research prompted us to examine the relationships among the NFkB pathway, miR-29s, and MMP-2 in vivo and ex vivo, with focus on an angiogenesis model, in other words experimental CNV.

\section{Materials and methods Materials}

The following substances, materials, and reagents (and suppliers) were used in this study: the miRCURY LNA ${ }^{\text {sm }}$ Universal RT microRNA PCR system including universal cDNA synthesis kit, SYBR ${ }^{\text {mi }}$ Green Master Mix, and the primers for has-miR-29s and 5 s RNA (Exiqon, Vedbaek, Denmark); Endofree Qiagen plasmid maxi kit (QIAGEN Gmbh, Hilden, Germany); Dual-Glo ${ }^{\text {Tm }}$ luciferase kit and FuGENE ${ }^{\text {mo }}$ HD transfection reagent, pGEM-T vector (Promega Branch, Beijing, China); Ex Taq and restriction enzymes (Takara, Dalian, China); Lipofectamine ${ }^{\mathrm{Tx}} 2000$ transfection reagent, Trizol, DNaseI (Invitrogen, Shanghai, China); miR-29a, b and c mimics (Ambion, Austin, Texas, United States); pMIR-REPORT ${ }^{\mathrm{mox}}$ vector (Applied Biosystems, Foster City, California, United States); 3-amino-9 ethylcarbazole (AEC) and VECTASTAIN Elite ABC kit (Vector Laboratories, Burlingame, California, United States); MMP-2 antibodies (Cell Signaling Technology, Beverly, Massachusetts, United States; Lifespan Biosciences, Atlanta, Georgia, United States); CD31 antibody (BD PharMingen, San Diego, California, United States); ARPE-19 and HEK 293 cells (ATCC, Manassas, Virginia, United States); Dulbecco's Modified Eagle Medium (DMEM), DMEM/F-12, Fetal Bovine Serum (FBS), Opti-MEM (Gibco, Gaithersburg, Maryland, United States); and Neg-50 frozen section media (Microm BioResource, Auburn, California, United States).

\section{Establishment of a CNV model}

$\mathrm{CNV}$ was induced in C57BL/6 mice at the age of between 6 and 8 weeks, with 6 burns surrounding the optic nerve by krypton red laser photocoagulation (50 $\mu \mathrm{m}$ spot size, 0.05 second duration, $260 \mathrm{~mW}$ ) as previously described $[24,25]$. The right eyes were treated with laser, and the left eyes were left untreated, serving as the control. A bubble formed at the laser spot, indicating rupture of the Bruch's membrane. The animals were euthanized between days 12 and 15 in order to assess the angiogenesis, and at days 3 and 5 for examining protein levels of $\mathrm{NFKB}_{\mathrm{K}}$ and miR-29s expression. The laser spots were evaluated for the presence of $\mathrm{CNV}$ using hematoxylin and eosin staining and immunohistochemistry against CD31. 


\section{Tissue preparation}

$\mathrm{CNV}$ was induced in mice as described above. The animals' eyes were enucleated at various time points. For immunohistochemistry analysis, eyes were imbedded in Neg-50 frozen section media and immediately frozen in liquid nitrogen. The samples were kept at $-80^{\circ} \mathrm{C}$ before cryostat sectioning. For western blot and reversetranscription (RT-qPCR) analyses of choroid-RPE tissue, the anterior segments, the vitreous, and the neurosensory retina of the eyes were removed, and the choroid-RPE layer was scraped from the sclera.

\section{Immunohistochemistry analysis}

The frozen eyeballs were cryosectioned at $10 \mu \mathrm{M}$ thickness. After fixation in acetone for 12 minutes at $4^{\circ} \mathrm{C}$ and blocking with goat serum, the cryosections were incubated overnight with primary antibody (CD31, 1:20; MMP-2, 1:100). The sections were then washed with $\mathrm{PBS}$ and subsequently incubated with biotinylated secondary antibody for $30 \mathrm{mi}-$ nutes at room temperature, followed by incubating with avidin-horseradish peroxidase complex (VECTASTAIN Elite $\mathrm{ABC}$ kit) for 30 minutes at ambient temperature. AEC was used to detect the peroxidase activity, after which the sections were counterstained with hematoxylin.

\section{Cell culture, treatment and transfection}

ARPE-19 cells were cultured in DMEM/F-12 supplemented to $10 \%$ with FBS. EA hy 926 and HEK-293 cells were cultured in DMEM supplemented to $10 \%$ with FBS. The cultures were maintained in a humidified 5\% $\mathrm{CO}_{2}$ incubator at $37^{\circ} \mathrm{C}$. Cells were passaged by use of 0.05\% trypsin/ Ethylenediaminetetraacetic acid (EDTA) (Life Technologies, Gaithersburg, United States). Before transfection, the culture media for ARPE-19 cells were switched to DMEM/F12 supplemented to $1 \%$ with FBS for 2 hours. Then, ARPE-19 cells were transfected with $50 \mathrm{nM}$ NFkB decoy oligodeoxynucleotides (ODN) or scrambled ODN with FuGENE ${ }^{\mathrm{mi}}$ HD transfection reagent and incubated for another 4.5 hours at $37^{\circ} \mathrm{C}$. The cultures were treated with tumor necrosis factor alpha (TNF- $\alpha$ ) $(10 \mathrm{ng} / \mathrm{mL})$ for 12 hours at $37^{\circ} \mathrm{C}$ and harvested for RT-qPCR analysis.

\section{Western blot analysis}

For detecting secreted MMP-2 contents from supernatants with immunoblotting, the conditioned media were collected and concentrated with an Eppendorf concentrator 5301 (Eppendorf AG, Hamburg, Germany). The loading volumes in SDS-PAGE gels from control and treated conditions were adjusted so that the loading from each condition originated from equal protein mass in culture dishes [26].

For MMP-2 immunoblotting, ARPE-19 and EA hy926 cells were seeded onto six-well plates and transfected with miR-29s mimics $(50 \mathrm{nM})$ using Lipofectamine ${ }^{\mathrm{Tm}} 2000$ in Opti-MEM media, according to the manufacturer's instructions. At 4 to 6 hours after transfection, the cells were switched to DMEM/F12 supplemented to $10 \%$ with FBS for another 48 hours and collected for analyses. The MMP-2 antibody (1:1000) and the peroxidase-conjugated secondary antibody (1:5000) were used, followed by enhanced chemiluminescence (ECL) detection.

For NFKB analysis, choroidal-RPE tissues were homogenized with protein lysis buffer containing protease inhibitor cocktail and centrifuged to remove insoluble material. The blotting procedures followed the protocol previously described [27].

\section{Reverse-transcription quantitative real-time PCR}

Total RNAs from choroidal-RPE tissue and cultured ARPE-19 cells were isolated using Trizol reagent. To prevent genomic contamination, RNA was further purified with DNase I (300 ng RNA was treated with $1 \mu \mathrm{L}$ DNase I at room temperature for 15 minutes and $65^{\circ} \mathrm{C}$ for 10 minutes after addition of $2.5 \mathrm{mM}$ EDTA). RNA (60 ng) was used to synthesize the cDNA with a cDNA synthesis kit (Exiqon) with deoxy-thymine nucleotide oligomer (oligo-dT) as the primer. The cDNA product (4 ng) was used as a template for PCR reaction with locked nucleic acid $\left(\mathrm{LNA}^{\mathrm{mm}}\right)$ primer sets (Exiqon) targeting has-miR-29s and $5 \mathrm{~s}$ was used as an internal reference gene. The miRCURY LNA ${ }^{\mathrm{ma}}$ SYBR Green was used as reaction dye, and all PCR reactions occurred in a 96well ABI plate format in $\mathrm{ViiA}^{\text {mi }} 7$ real-time PCR system (Applied Biosystems). The relative quantification method (a delta-delta $\mathrm{C}(\mathrm{T})$ ) was adopted and 40 cycles of PCR reactions $\left(95^{\circ} \mathrm{C}\right.$ for 10 seconds and $60^{\circ} \mathrm{C}$ for 1 minute) were started with $50^{\circ} \mathrm{C}$ for 2 minutes, followed by $95^{\circ} \mathrm{C}$ for 10 minutes. Dissociative curves were used to confirm the reaction specificity.

\section{Plasmid construction and luciferase reporter assay}

The incorporation of 3'UTR of human MMP-2 into multiple cloning sites of PMIR-REPORT ${ }^{\mathrm{Tix}}$ vector involved two steps of cloning strategy: first, ligation of the 3'UTR PCR product into PGEM-T vector, named T1-MMP2-3'UTR, and second, introduction of new restriction sites on the PCR product, with T1-MMP-2-3'-UTR as the template. The PCR product was then subcloned into PMIRREPORT Tw $^{\text {Tu }}$. Briefly, the PCR product of the 3'UTR of the human MMP-2 was amplified from the genomic DNA of ARPE-19 cells with the forward primer: 5'-TTC CTC TCC ACT GCC TTC GAT A-3' and the reverse primer: 5'AGG ACA GAG GGA CTA GAG CTT A-3' corresponding to the sequences from 2319 to $2340 \mathrm{bp}$ and from 3257 to 3278 bp respectively, in the human MMP2 mRNA 3'-UTR (GenBank, NM_004530.4). The PCR began at an initial cycle $\left(94^{\circ} \mathrm{C}\right.$ for 30 seconds, $62^{\circ} \mathrm{C}$ for 30 seconds, 
and $72^{\circ} \mathrm{C}$ for 45 seconds), followed by serial cycles with a Tm stepdown of $1^{\circ} \mathrm{C}$ per cycle until $\mathrm{Tm}=52^{\circ} \mathrm{C}$ was reached, and the cycle was then repeated 25 times. A total of 35 reaction cycles were performed with Takara LA Taq (Takara), preceded by a denaturation of 4 minutes at $94^{\circ} \mathrm{C}$ and finished with a 10 -minute extension at $72^{\circ} \mathrm{C}$. After confirming the presence of PCR product with sequencing, the regular PCR method (instead of touchdown PCR) was adopted with the forward primer: CATG $A C T$ AGT CCT CTC CAC TGC CTT CGA TA (SpeI restriction site is indicated italic) and the reverse primer: CATG AAG CTT AGG ACA GAG GGA CTA GAG CT (HindIII restriction site is indicated italic). A total of 30 reactions were conducted $\left(94^{\circ} \mathrm{C}\right.$ for 30 seconds, $52^{\circ} \mathrm{C}$ for 30 seconds, and $72^{\circ} \mathrm{C}$ for 45 seconds). The PCR reactions were concluded with a 10 -minute extension at $72^{\circ} \mathrm{C}$. The resultant vector was named as PMIR-MMP-2 3'UTR.

HEK-293 cells were transfected in serum-free DMEM into 24-well plates with $50 \mathrm{ng}$ pMIR-MMP-2 3'UTR containing firefly luciferase coding sequence, $25 \mathrm{ng}$ pRL-SV40 Renilla vector (Promega Branch, Beijing, China) and $5 \mathrm{nM}$ miR-29 mimics or negative control mimics (NC). The plasmid mixtures were incubated with Lipofectamine ${ }^{\mathrm{Tx}}$
2000 in opti-MEM for 30 minutes, transfected to the HEK-293 cells for 3 hours, washed away with DMEM, and then switched to regular culture media, DMEM plus 10\% FBS. At 24 hours after transfection, Firefly and Renilla luciferase activities were measured with a dual luciferase reporter assay kit (Promega Branch, Beijing, China) according to the manufacturer's instructions.

\section{Decoy oligodeoxynucleotide (ODN)}

Double-stranded ODNs used as NFKB decoy were synthesized as previously described [28]. The sequences were 5'-CCT TGA AGG GAT TTC CCT CC -3' and 3'-G GA GGG AAA TCC CTT CAA GG-5'. Scramble ODNs served as control decoy, and the sequences were 5'-TTG CCG TAC CTG ACT TAG CC-3' and 3'-AAC GGC ATG GAC TGA ATC GG-5’.

\section{Statistical analysis}

Data were analyzed for significant difference $(P<0.05)$ with a one way analysis of variance (ANOVA) and Bonferroni post hoc test for multiple comparisons (SPSS 15.0.1; SPSS Inc., Chicago, Illinois, United States).

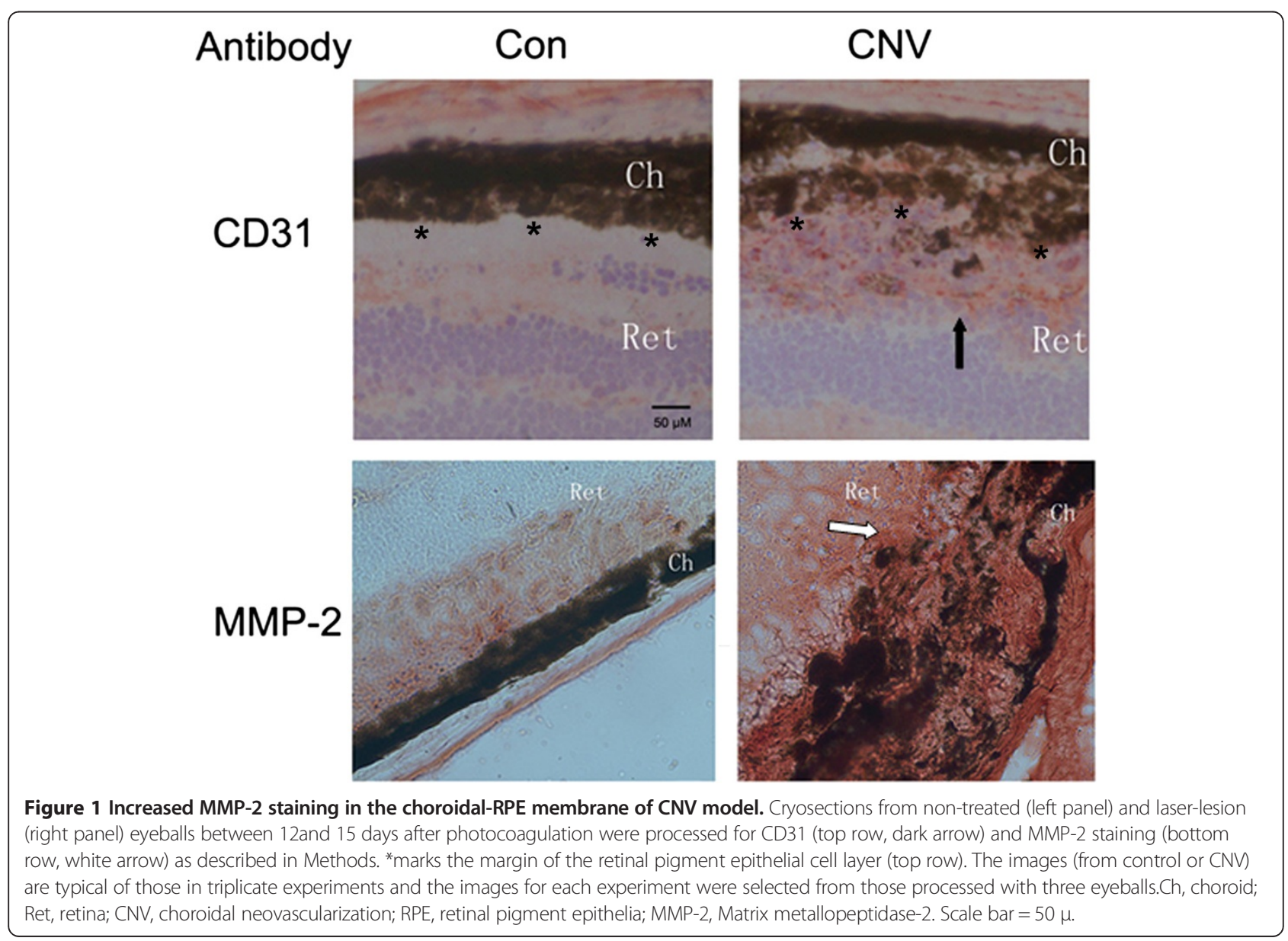




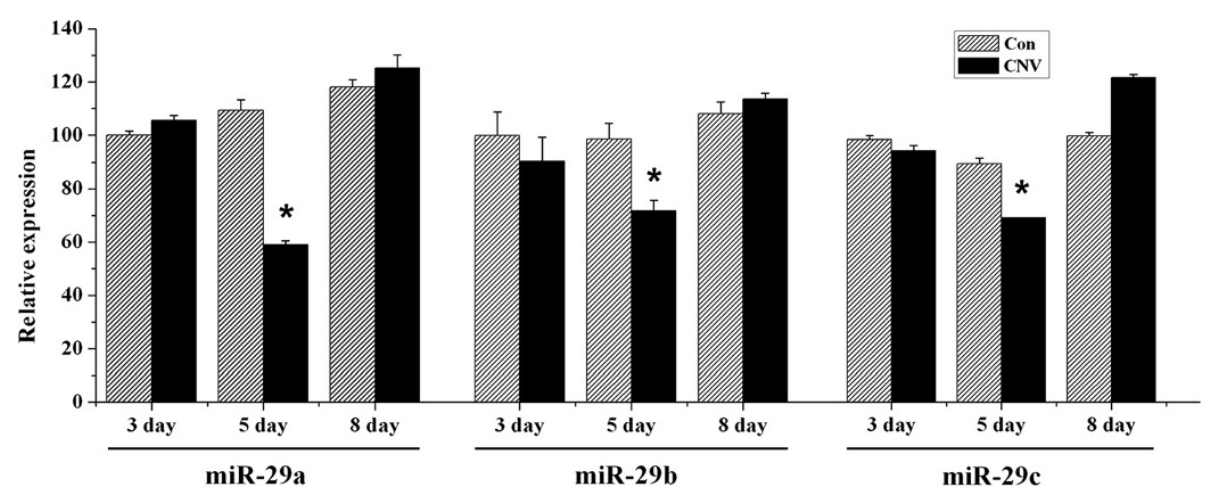

Figure 2 Decreased miR-29 s level in choroidal-RPE tissue of CNV. Choroidal-RPE tissues were isolated from non-treated and laser-treated eyeballs at days three, five and eight after photocoagulation and processed for miR-29s detection with RT-qPCR, as described in Methods. The values of control were set as $100 \%$ and the results from treatments were normalized to the control values. The results shown are mean ( \pm SEM) averaged from three independent experiments with tissues for each experiment collected from between 5 and 7 eyeballs. ${ }^{*} P<0.05$ versus control. CNV, choroidal neovascularization; RPE, retinal pigment epithelia.

\section{Results}

Increased CNV and MMP-2 staining in the choroid-RPE membrane of the CNV model

Krypton laser photocoagulation was used to create $\mathrm{CNV}$ in the right eyes of six to eight week-old mice; the left eyes of the mice were used as controls. At 12 to 15 days after fundus photocoagulation, the cryosections from serial sections of the laser-treated eyes were examined with immunohistochemistry against CD31, an endothelial marker of blood vessels. The RPE layer was destroyed and CD31 staining was present in the subretinal space in the $\mathrm{CNV}$ group, indicating that laser treatment destroyed the choroid-RPE barrier at the laser spots and induced endothelial cells to migrate to the subretinal space, which is evidence that successful CNV was created (Figure 1 top row, right panel). To demonstrate the roles of MMP-2 in the process, the serial sections from the same sets of cryosections were subjected to MMP-2 staining; increased staining was detected in the choroid-RPE membrane (Figure 1 bottom row, right panel).

\section{Decreased miR-29s in choroid-RPE tissue of the CNV model} Although miRNAs are involved in the complicated network of angiogenic regulation, the roles of miRNAs in the development of CNV are rarely characterized. Bioinformatic analyses with miRBase, Target Scan, and Pic Tar indicated that MMP-2 regulation could be mediated by miR-29s, and evidence indicated that miR-29b is involved in tumor angiogenesis, invasion, and metastasis by mediating MMP-2 protein expression [15]. Therefore, we decided to search for miR-29s in choroid-RPE tissue from the CNV model as a means of determining the possible roles of miR-29s in CNV. Since the amount of the choroidal-RPE tissue is far too low for northern blot detection, we used RT-qPCR to quantify the miR-29s. The linear amplification of RT-qPCR was tested with

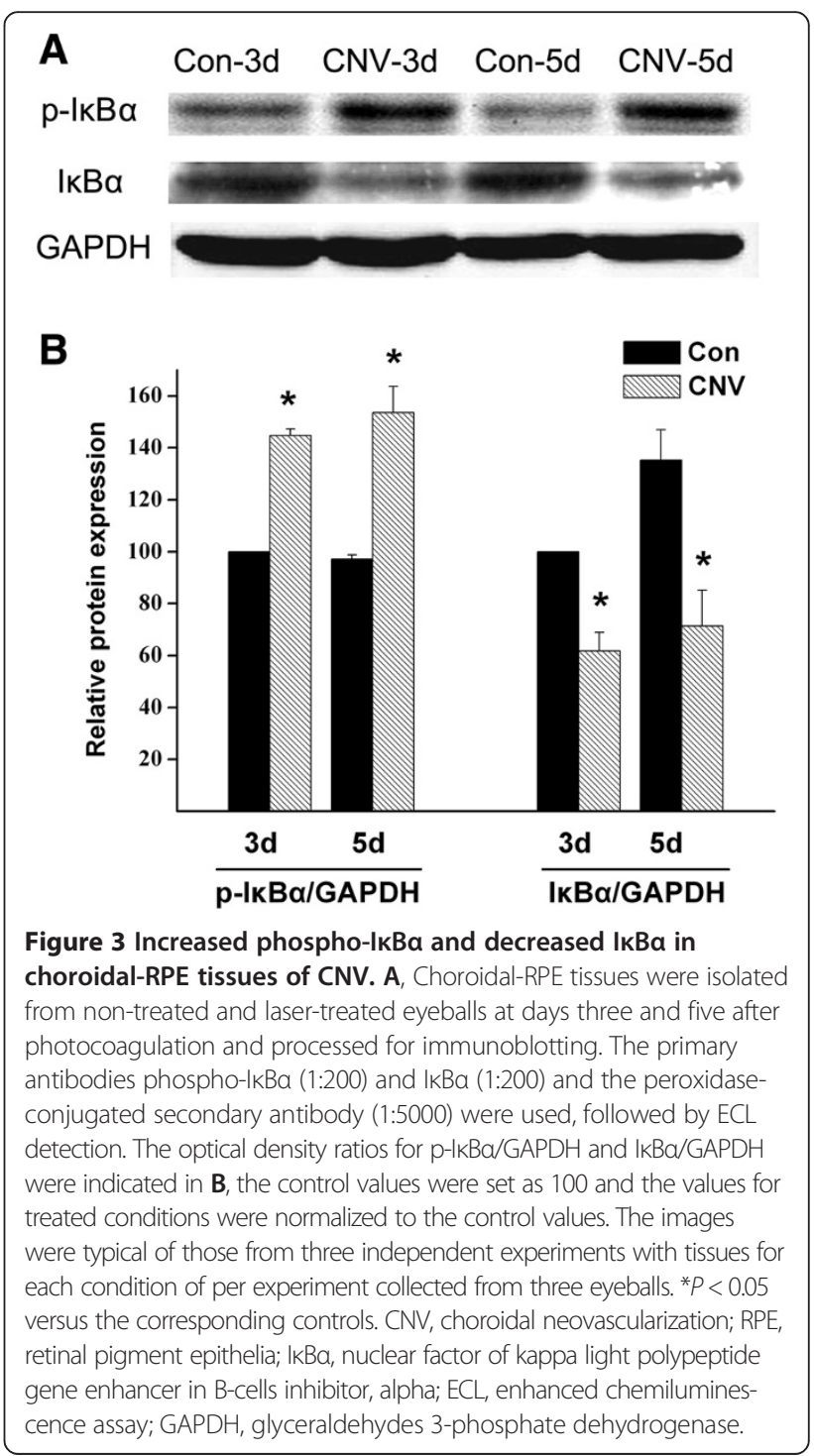


amplication of miR-29s, using various amounts of mouse cerebral tissues with weight differences in order (data not shown). Interestingly, we found that miR-29s including miR-29a, b, and c, at five days after photocoagulation, decreased significantly compared to the amounts in the contralateral eyes whereas at early (three days) or later time points (eight days), the miR-29s had not changed (Figure 2).

\section{NFkB activation in choroid-RPE tissue of the CNV model}

Recent studies suggested that the development of $\mathrm{CNV}$ is significantly suppressed by inhibiting the activation of NFкB $[22,29,30]$. NFkB complex is sequestered by inhibitors ( $\mathrm{I} \mathrm{K}$ family proteins in cytoplasm in an inactive state) but phosphorylated by $I_{\kappa} B$ kinase [31] under stimuli (phospho-IkB is subjected to degradation by proteasome complex). As a consequence, $\mathrm{NF}_{\mathrm{K} B}$ is released from $\mathrm{I} \kappa \mathrm{B}$ binding to enter the nucleus and activate transcription of specific genes. To determine whether NFkB was activated during the development of $\mathrm{CNV}$, we analyzed phospho$\mathrm{I} \kappa \mathrm{B} \alpha$ and $\mathrm{I} \kappa \mathrm{B} \alpha$ [32]. Western blot detected increased phospho-IkB $\alpha$ and decreased IkB $\alpha$ in choroid-RPE tissue of CNV model at days three and five after laser induction, compared with amounts in non-treated eyes, a finding suggesting that the laser treatment had activated the NFKB pathway in the choroid-RPE tissue (Figure 3).

It was recently shown that the transcription factor $\mathrm{NF} K \mathrm{~B}$ negatively regulates $\mathrm{miR}-29 \mathrm{~b} / \mathrm{c}$ in various cells $[18,23]$. We therefore examined the possible role of this pathway in the regulation of miR-29s in ARPE-19 cells, a human RPE cell line. Previous studies indicated that TNF $\alpha$ is significantly increased in choroidal-RPE tissue in laser-induced neovascularization mice model $[33,34]$ and contributes to laser-induced CNV formation [35,36]. Consequently, we tested the possible regulatory effect of TNF $\alpha$ on the miR-29 family and determined stimulation with TNF $(10 \mathrm{ng} / \mathrm{mL})$ resulted in significant downregulation of all miR-29 members (Figure 4). Furthermore, transfection of the synthetic $\mathrm{NFkB}$ decoy, imitating the NFkB binding site that has been reported to inhibit NFKB activation [28], rescued the down-regulation of miR-29s by TNF $\alpha$ (Figure 4). These results were consistent with a previous study indicating that a chemical inhibitor of NFKB activation increases the expression of miR-29s [37].

\section{miR-29s down-regulated MMP-2 expression in RPE and endothelial cells}

In choroidal-RPE tissue of the CNV model, the increased expression of the MMP-2 was accompanied with decreased expression of miR-29s (Figure 1 and Figure 2). Further bioinformatic analysis reveals a complementary sequence for all the miR-29 members in the 3'-UTR of MMP-2 (data not shown). We subsequently investigated the possibility that miR-29s down-regulate MMP-2 expression in vitro. Since RPE and endothelial cells are the major cell types secreting MMP-2 in choroid-RPE tissue, ARPE-19 and EAhy 926 were used to test whether the expression of MMP-2 was regulated by miR-29s. We transfected both

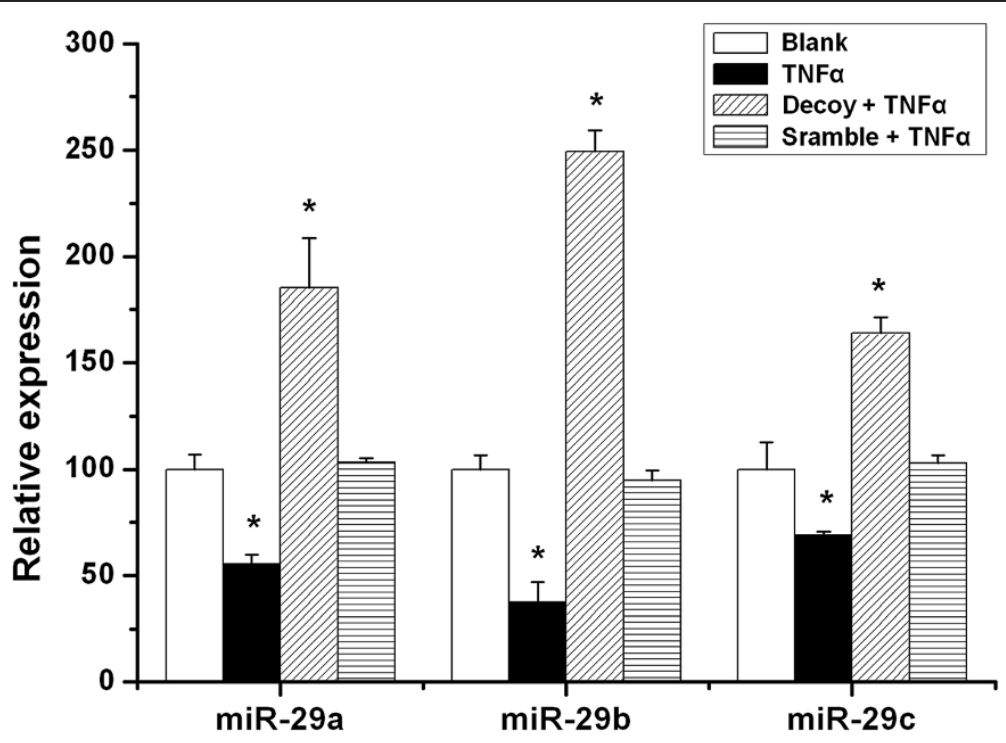

Figure 4 TNFa reduced miR-29 s, which was reversed by NFKB decoy. ARPE-19 cells were cultured in six-well dishes and transfected with NFKB decoy ODNs (50 nM) or scramble ODNs (50 nM) when the cell densities reached approximately $70 \%$ confluency. The ARPE- 19 cells were then treated with TNFa $(10 \mathrm{ng} / \mathrm{mL})$ for 12 hours and miR-29 s levels were determined by RT-qPCR. The values of control were set as $100 \%$ and the results from treatments were normalized to control. The results shown are mean ( \pm SEM) from at least three independent experiments with each condition per experiment from quadruplicate cultures. ${ }^{*} P<0.05$ versus control. NFKB, nuclear factor of kappa light polypeptide gene enhancer in B-cells; ODN, oligodeoxynucleotide; TNFa, tumor necrosis factor alpha. 
types of cells with miR-29 mimics or non-specific control miRNA mimics (NC mimic). Transfection with individual miR-29 reduced protein levels of MMP-2 to a similar level, but transfection with miR-29 b/c induced a larger decrease in EA hy926 than did transfection with miR-29a (Figure 5A and B).

To confirm whether 3'-UTR of MMP-2 is a functional target of miR-29s, we cloned a reporter plasmid containing the 3'UTR of MMP-2 (pMIR-MMP-2 3'UTR, Methods) between the firefly luciferase reporter gene and polyA tail in pMIR-REPORT ${ }^{\mathrm{Tm}}$. HEK-293 cells, which contain low levels of endogenous miR-29s, were transfected with individual miR-29a, b, and c mimics, pMIRMMP-2 3'UTR, and Renilla luciferase reporter plasmids. The luciferase assay indicated that co-transfection of miR-29a and b mimics resulted in significantly decreased luciferase activity, whereas transfection of miR-29c mimic had a limited effect (Figure 5C).

\section{Discussion}

Our results in the $\mathrm{CNV}$ model demonstrated that NFKB activation accompanied decreased miR-29s levels in choroidal-RPE tissue, which contained elevated levels of MMP-2 protein. At the cellular level, TNF $\alpha$, an important cytokine in laser-induced $\mathrm{CNV}$, decreased miR-29s in ARPE-19 cells. This effect was dependent on NFkB pathway activation. Finally, we confirmed that miRNA-29s downregulated MMP-2 expression in the RPE and endothelial cells. Considering the critical roles of RPE and choroidal endothelial cells in angiogenic processes, our results suggested that NFKB-miR-29s-MMP-2 circuitry contributes to the angiogenesis in $\mathrm{CNV}$.

Co-transfection with the $\mathrm{NF}_{\mathrm{K} B}$ decoy rescued the reduction of miR-29s by TNF $\alpha$, and the effects exceeded the control level, possibly due to the complete blockade of NFkB pathway by the decoy (Figure 4). The miR-29s mimics $a$ and $b$ reduced secreted MMP-2 levels to the same extent as in ARPE-19 cells which is in contrast to the differential effects in EA hy926 and probably reflects the cell-type specificity of miRNA-29s (Figure 5). For instance, as reported, epigenetic modification by miR-29b is distinct from miR-29a and c [38], whereas miR-29a specifically modulates the angiogenic process in endothelial cells [39].

Although we demonstrated the possible roles of NFkBmiR-29s-MMP2 circuitry in CNV, the involved molecules and the associated regulatory mechanisms in $\mathrm{CNV}$ are versatile. For instance, $\mathrm{NF \kappa B}$ pathway activation is associated with angiogenesis, and blockade of the NFkB pathway inhibits angiogenesis in various contexts [21,22]. One defined mechanism of MMP-2 regulation by NFKB activation is that the increasing transcription of MT1MMP $[40,41]$ cleaves pro-MMP-2 to its mature form, MMP-2 [42] (the pathway is independent of miR-29s).
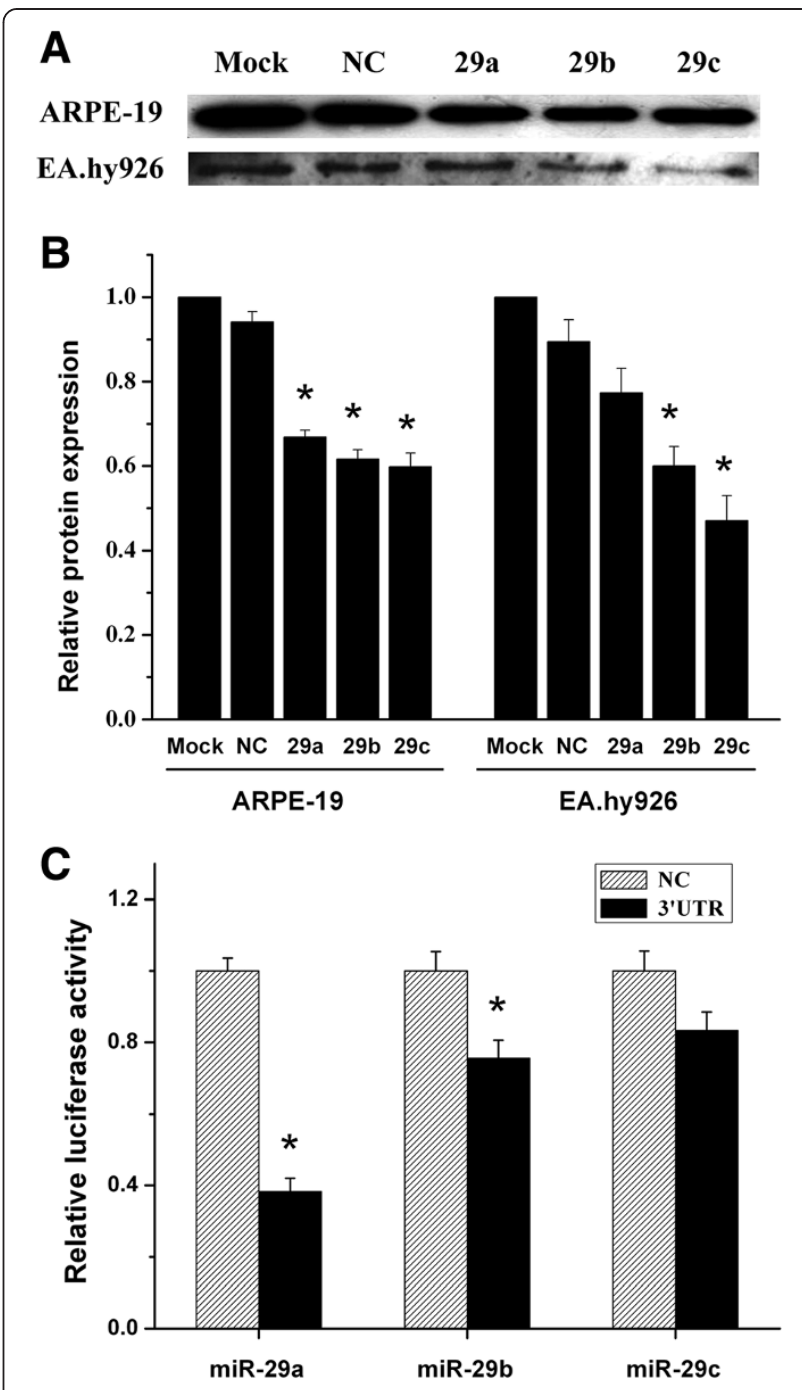

Figure 5 miR-29 s down-regulated MMP-2 in ARPE-19 and EA hy926 cells. A, ARPE-19 or EA hy926 cells were cultured in six-well dishes and transfected with miR-29a, b, and c (50 nM), and NC $(50 \mathrm{nM})$ individually when the cell densities reached approximately $70 \%$ confluency. After being cultured for 48 hours, the supernatants were harvested for examining the MMP-2 contents with western blot as described in Methods. The images shown are typical of those from three independent experiments with each condition from triplicate cultures. B, The ratios of Mock between supernatant MMP-2 and protein mass in culture wells were set as 1 and the values for treated conditions were normalized to the control values. C, HEK-293 cells were cultured in 96-well dishes, and each well was transfected with 50 ng pMIR-MMP-2 3'UTR/firefly luciferase, 25 ng pRL-SV40 Renilla luciferase vector, and $5 \mathrm{nM}$ miR-29 mimics or $5 \mathrm{nM} \mathrm{NC}$. Luciferase activities were determined 24 hours after transfection and the results were expressed as the ratios between the activity of firefly luciferase and that of Renillaluciferase. The results shown were mean $( \pm$ SEM) from three independent experiment with each condition per experiment from quadruplicate cultures. The values of control were set as 1 and the results from treatments were normalized to control. ${ }^{*} P<0.05$ versus NC. NC, negative control; MMP-2, matrix metallopeptidase-2; UTR, untranslated region. 
However, overproduced MMP2, either from direct activation of $\mathrm{NFKB}$ or the reduced level of miR-29s in various contexts, leads to enhanced degradation of the basement membrane and extracellular matrix, which are prerequisites for endothelial sprout invasion, an early event in angiogenesis [5]. In addition to the MMP family, including MMP-2 and MMP-9 [25] which are involved in CNV, the angiogenic process in $\mathrm{CNV}$ is complicated, involving a network of interactions between RPE cells and choroidal endothelial cells. These paracrine interactions were reportedly mediated by molecules secreted by either kind of cell, for example, the molecules related to the activation of the plasminogen/plasminogen system [40], angiogenic factor, VEGF $[4,10]$, cytokine, TNF $\alpha$ [33], and monocyte chemotactic protein1 (MCP1) produced by invading macrophages at CNV sites [43].

It is clear that MMP-2 plays an essential role in regulating the angiogenesis in $\mathrm{CNV}$, an opinion supported by the observations that CNV is less in MMP-2 deficient mice [8] and is decreased by anti-angiogenic approaches, including the use of MMP inhibitors targeting MMP-2, 9 and MT1-MMP [25]. Here, we demonstrated a possible MMP2 regulatory pathway in vivo and ex vivo in CNV via down-regulation of miR-29s mediated by NFkB activation. These results suggest a promising strategy for resolving CNV by targeting miR-29s levels.

\section{Abbreviations \\ AMD: Age-related macular degeneration; CNV: Choroidal neovascularization; miR-29: microRNA 29; HUVEC: Human umbilical vein endothelial cell; MMP-2: Matrix metallopeptidase-2; MT1-MMP: Membrane type1 metalloprotease; NFKB: Nuclear factor kappa-like-chain-enhancer of activated B cells; ODN: Oligodeoxynucleotide; RPE: Retinal pigment epithelial cell; RT-qPCR: Reverse transcription quantitative real-time PCR; TIMP: Tissue inhibitors of metalloproteinase; TNFa: Tumor necrosis factor alpha; VEGF: Vascular endothelial growth factor; UTR: untranslated region.}

\section{Competing interests}

The authors declare that they have no competing interests.

\begin{abstract}
Authors' contributions
CJ performed RT-qPCR, cloned MMP-2 luciferase assay reporter, did luciferase assay and western blot, analyzed the data, and contributed to the writing. YGB performed western blot, immunohistochemistry and miR-29s northern blot, and joined in the luciferase reporter cloning. LB created the CNV model. XW helped the operation during the procedure of laser photocoagulation. $L X L$ provided CNV facilities. CXY and YDS provided technique support on miR-29s northern blot. Shan $\mathrm{G}$ and Qu J provided expert opinions on the projects. WS conceived of the project, designed the experiments, provided technique support, analyzed the data, and wrote the manuscript. All authors read and approved the final manuscript.
\end{abstract}

\section{Acknowledgements}

The authors thank our colleague, Professor Hou Ling, for providing the ARPE-19 cell line, which was obtained from the American Type Culture Collection (ATCC). The study was supported by the National Science Foundation of China (NSFC, 81371027), by Qianjiang Scholar Scheme (QJD1202020), by Chinese Ministry of Education (20133321120002), and by start-up funding (89210001) from Wenzhou Medical University to Dr Shengzhou Wu. It was also supported by the National Natural Science Foundation of China (81171074) to Dr Ge Shan; supported by the ZheJiang Province Natural Science Foundation (LQ14C060002) and start-up funding (YNCX201310) from Wenzhou Medical University to DrJingjingCai.

\section{Author details}

'School of Optometry and Ophthalmology and Eye Hospital, Wenzhou Medical University, 270 Xueyuan Road, Wenzhou, Zhejiang 325003, People's Republic of China. ${ }^{2}$ State Key Laboratory Cultivation Base and Key Laboratory of Vision Science, Ministry of Health, China and Zhejiang Provincial Key Laboratory of Ophthalmology and Optometry, 270 Xueyuan Road, Wenzhou, Zhejiang 325003, People's Republic of China. ${ }^{3}$ School of Life Sciences \& CAS Key Laboratory of Brain Function and Disease, University of Science and Technology of China, 443 Huangshan Road, Hefei, Anhui Province 230027, People's Republic of China.

Received: 9 December 2013 Accepted: 1 May 2014

Published: 15 May 2014

\section{References}

1. Bashshur ZF, Bazarbachi A, Schakal A, Haddad ZA, El Haibi CP, Noureddin $B N$ : Intravitreal bevacizumab for the management of choroidal neovascularization in age-related macular degeneration. Am J Ophthalmol 2006, 142:1-9.

2. Hayasaka S, Uchida M, Setogawa T: Subretinal hemorrhages with or without choroidal neovascularization in the maculas of patients with pathologic myopia. Graefes Arch Clin Exp Ophthalmol 1990, 228:277-280.

3. Yoshida T, Ohno-Matsui K, Ohtake Y, Takashima T, Futagami S, Baba T, Yasuzumi K, Tokoro T, Mochizuki M: Long-term visual prognosis of choroidal neovascularization in high myopia: a comparison between age groups. Ophthalmology 2002, 109:712-719.

4. Kwak N, Okamoto N, Wood JM, Campochiaro PA: VEGF is major stimulator in model of choroidal neovascularization. Invest Ophthalmol Vis Sci 2000, 41:3158-3164

5. Stetler-Stevenson WG: Matrix metalloproteinases in angiogenesis: a moving target for therapeutic intervention. J Clin Invest 1999, 103:1237-1241.

6. Wilcock DM, Morgan D, Gordon MN, Taylor TL, Ridnour LA, Wink DA, Colton CA: Activation of matrix metalloproteinases following anti-Abeta immunotherapy; implications for microhemorrhage occurrence. J Neuroinflammation 2011, 8:115.

7. Majka S, McGuire P, Colombo S, Das A: The balance between proteinases and inhibitors in a murine model of proliferative retinopathy. Invest Ophthalmol Vis Sci 2001, 42:210-215.

8. Berglin L, Sarman S, van der Ploeg I, Steen B, Ming Y, Itohara S, Seregard S, Kvanta A: Reduced choroidal neovascular membrane formation in matrix metalloproteinase-2-deficient mice. Invest Ophthalmol Vis Sci 2003, 44:403-408.

9. Ohno-Matsui K, Uetama T, Yoshida T, Hayano M, Itoh T, Morita I, Mochizuki M: Reduced retinal angiogenesis in MMP-2-deficient mice. Invest Ophthalmol Vis Sci 2003, 44:5370-5375.

10. Spilsbury K, Garrett KL, Shen WY, Constable IJ, Rakoczy PE: Overexpression of vascular endothelial growth factor (VEGF) in the retinal pigment epithelium leads to the development of choroidal neovascularization. Am J Pathol 2000, 157:135-144

11. Hoffmann S, He S, Ehren M, Ryan SJ, Wiedemann P, Hinton DR: MMP-2 and MMP-9 secretion by RPE is stimulated by angiogenic molecules found in choroidal neovascular membranes. Retina 2006, 26:454-461.

12. Chen K, Rajewsky N: The evolution of gene regulation by transcription factors and microRNAs. Nat Rev Genet 2007, 8:93-103.

13. Mourelatos Z, Dostie J, Paushkin S, Sharma A, Charroux B, Abel L, Rappsilber J, Mann M, Dreyfuss G: miRNPs: a novel class of ribonucleoproteins containing numerous microRNAs. Genes Dev 2002, 16:720-728.

14. Fabbri M, Garzon R, Cimmino A, Liu Z, Zanesi N, Callegari E, Liu S, Alder H, Costinean S, Fernandez-Cymering C, Volinia S, Guler G, Morrison CD, Chan KK, Marcucci G, Calin GA, Huebner K, Croce CM: MicroRNA-29 family reverts aberrant methylation in lung cancer by targeting DNA methyltransferases 3A and 3B. Proc Natl Acad Sci U S A 2007, 104:15805-15810.

15. Fang JH, Zhou HC, Zeng C, Yang J, Liu Y, Huang X, Zhang JP, Guan XY, Zhuang SM: MicroRNA-29b suppresses tumor angiogenesis, invasion, and metastasis by regulating matrix metalloproteinase 2 expression. Hepatology 2011, 54:1729-1740.

16. Hebert SS, Horre K, Nicolai L, Papadopoulou AS, Mandemakers W, Silahtaroglu AN, Kauppinen S, Delacourte A, De Strooper B: Loss of microRNA cluster miR-29a/b-1 in sporadic Alzheimer's disease correlates with increased BACE1/beta-secretase expression. Proc Natl Acad Sci U S A 2008, 105:6415-6420. 
17. Park SY, Lee JH, Ha M, Nam JW, Kim VN: miR-29 miRNAs activate p53 by targeting p85 alpha and CDC42. Nat Struct Mol Biol 2009, 16:23-29.

18. Wang H, Garzon R, Sun H, Ladner KJ, Singh R, Dahlman J, Cheng A, Hall BM, Qualman SJ, Chandler DS, Croce CM, Guttridge DC: NF-kappaB-YY1-miR-29 regulatory circuitry in skeletal myogenesis and rhabdomyosarcoma. Cancer Cell 2008, 14:369-381.

19. Ugalde AP, Ramsay AJ, de la Rosa J, Varela I, Marino G, Cadinanos J, Lu J, Freije JM, Lopez-Otin C: Aging and chronic DNA damage response activate a regulatory pathway involving miR-29 and p53. EMBO J 2011, 30:2219-2232.

20. Kiriakidis S, Andreakos E, Monaco C, Foxwell B, Feldmann M, Paleolog E: VEGF expression in human macrophages is NF-kappaB-dependent: studies using adenoviruses expressing the endogenous NF-kappaB inhibitor IkappaBalpha and a kinase-defective form of the lkappaB kinase 2. J Cell Sci 2003, 116:665-674.

21. Huang S, Pettaway CA, Uehara H, Bucana CD, Fidler IJ: Blockade of NF-kappaB activity in human prostate cancer cells is associated with suppression of angiogenesis, invasion, and metastasis. Oncogene 2001, 20:4188-4197.

22. Jo N, Ogata N, Aoki M, Otsuji T, Morishita R, Kaneda Y, Matsumura M: Effective transfection of a cis element "decoy" of the nuclear factor-kappaB binding site into the experimental choroidal neovascularization. Curr Eye Res 2002, 24:465-473.

23. Mott $J$, Kurita S, Cazanave SC, Bronk SF, Werneburg NW, Fernandez-Zapico ME: Transcriptional suppression of mir-29b-1/mir-29a promoter by c-Myc, hedgehog, and NF-kappaB. J Cell Biochem 2010, 110:1155-1164.

24. Bora PS, Hu Z, Tezel TH, Sohn JH, Kang SG, Cruz JM, Bora NS, Garen A, Kaplan $\mathrm{HJ}$ : Immunotherapy for choroidal neovascularization in a laser-induced mouse model simulating exudative (wet) macular degeneration. Proc Natl Acad Sci U S A 2003, 100:2679-2684

25. Lambert V, Wielockx B, Munaut C, Galopin C, Jost M, Itoh T, Werb Z, Baker A, Libert C, Krell HW, Foidart JM, Noël A, Rakic JM: MMP-2 and MMP-9 synergize in promoting choroidal neovascularization. FASEB J 2003, 17:2290-2292.

26. Lokeshwar VB, Cerwinka WH, Lokeshwar BL: HYAL1 hyaluronidase: a molecular determinant of bladder tumor growth and invasion. Cancer Res 2005, 65:2243-2250.

27. Jiang H, Fang J, Wu B, Yin G, Sun L, Qu J, Barger SW, Wu S: Overexpression of serine racemase in retina and overproduction of $D$-serine in eyes of streptozotocin-induced diabetic retinopathy. J Neuroinflammation 2011, 8:119.

28. Son G, limuro Y, Seki E, Hirano T, Kaneda Y, Fujimoto J: Selective inactivation of NF-kappaB in the liver using NF-kappaB decoy suppresses CCl4-induced liver injury and fibrosis. Am J Physiol Gastrointest Liver Physiol 2007, 293:G631-G639.

29. Izumi-Nagai K, Nagai N, Ohgami K, Satofuka S, Ozawa Y, Tsubota K Umezawa K, Ohno S, Oike Y, Ishida S: Macular pigment lutein is antiinflammatory in preventing choroidal neovascularization. Arterioscler Thromb Vasc Biol 2007, 27:2555-2562.

30. Izumi-Nagai K, Nagai N, Ohgami K, Satofuka S, Ozawa Y, Tsubota K, Ohno S, Oike $Y$, Ishida S: Inhibition of choroidal neovascularization with an anti-inflammatory carotenoid astaxanthin. Invest Ophthalmol Vis Sci 2008 49:1679-1685.

31. Mustafa AK, Ahmad AS, Zeynalov E, Gazi SK, Sikka G, Ehmsen JT, Barrow RK, Coyle JT, Snyder SH, Dore S: Serine racemase deletion protects against cerebral ischemia and excitotoxicity. J Neurosci 2010, 30:1413-1416.

32. Hayden MS, Ghosh S: Shared principles in NF-kappaB signaling. Cell 2008, 132:344-362.

33. Shi X, Semkova I, Muther PS, Dell S, Kociok N, Joussen AM: Inhibition of TNF-alpha reduces laser-induced choroidal neovascularization. Exp Eye Res 2006, 83:1325-1334.

34. Shi X, Semkova I, Kociok N, Gavranic C, Becker M, Joussen AM, Kirchhof B: Effect of anti-TNF-alpha on laser-induced choroidal neovascularization. Zhonghua Yan Ke Za Zhi 2008, 44:200-206.

35. Lichtlen $\mathrm{P}$, Lam TT, Nork TM, Streit T, Urech DM: Relative contribution of VEGF and TNF-alpha in the cynomolgus laser-induced CNV model: comparing the efficacy of bevacizumab, adalimumab, and ESBA105. Invest Ophthalmol Vis Sci 2010, 51:4738-4745.

36. Jasielska M, Semkova I, Shi X, Schmidt K, Karagiannis D, Kokkinou D, Mackiewicz J, Kociok N, Joussen AM: Differential role of tumor necrosis factor (TNF)-alpha receptors in the development of choroidal neovascularization. Invest Ophthalmo/ Vis Sci 2010, 51:3874-3883.
37. Roderburg C, Urban GW, Bettermann K, Vucur M, Zimmermann H, Schmidt S, Janssen J, Koppe C, Knolle P, Castoldi M, Tacke F, Trautwein C, Luedde T: Micro-RNA profiling reveals a role for miR-29 in human and murine liver fibrosis. Hepatology 2011, 53:209-218.

38. Chen KC, Wang YS, Hu CY, Chang WC, Liao YC, Dai CY, Juo SH: OxLDL up-regulates microRNA-29b, leading to epigenetic modifications of MMP-2/MMP-9 genes: a novel mechanism for cardiovascular diseases. FASEB J 2011, 25:1718-1728.

39. Yang Z, Wu L, Zhu X, Xu J, Jin R, Li G, Wu F: MiR-29a modulates the angiogenic properties of human endothelial cells. Biochem Biophys Res Commun 2013, 434:143-149.

40. Han YP, Tuan TL, Wu H, Hughes M, Garner WL: TNF-alpha stimulates activation of pro-MMP2 in human skin through NF-(kappa) B mediated induction of MT1-MMP. J Cell Sci 2001, 114:131-139.

41. Philip S, Bulbule A, Kundu GC: Osteopontin stimulates tumor growth and activation of promatrix metalloproteinase-2 through nuclear factor-kappa B-mediated induction of membrane type 1 matrix metalloproteinase in murine melanoma cells. J Biol Chem 2001, 276:44926-44935.

42. Deryugina El, Ratnikov B, Monosov E, Postnova TI, DiScipio R, Smith JW, Strongin AY: MT1-MMP initiates activation of pro-MMP-2 and integrin alphavbeta3 promotes maturation of MMP-2 in breast carcinoma cells. Exp Cell Res 2001, 263:209-223.

43. Sakurai E, Anand A, Ambati BK, van Rooijen N, Ambati J: Macrophage depletion inhibits experimental choroidal neovascularization. Invest Ophthalmol Vis Sci 2003, 44:3578-3585.

\section{doi:10.1186/1742-2094-11-88}

Cite this article as: Cai et al:: Roles of NFKB-miR-29s-MMP-2 circuitry in experimental choroidal neovascularization. Journal of Neuroinflammation 2014 11:88.

\section{Submit your next manuscript to BioMed Central and take full advantage of:}

- Convenient online submission

- Thorough peer review

- No space constraints or color figure charges

- Immediate publication on acceptance

- Inclusion in PubMed, CAS, Scopus and Google Scholar

- Research which is freely available for redistribution 